\title{
Self-Stigma and Treatment Effectiveness in Patients with SSRI Non-Responsive Obsessive-Compulsive Disorder
}

This article was published in the following Dove Press journal: Psychology Research and Behavior Management

\author{
Marie Ociskova $\mathbb{D}^{\prime}$ \\ Jan Prasko (D) ${ }^{1-3}$ \\ Jakub Vanek (D) \\ Michaela Holubova ${ }^{4}$ \\ Frantisek Hodny (D) \\ Klara Latalova' \\ Krystof Kantor $\mathbb{D}^{\prime}$ \\ Vlastimil Nesnidal (D) \\ 'Department of Psychiatry, Faculty of \\ Medicine and Dentistry, Palacky \\ University in Olomouc, University \\ Hospital in Olomouc, Olomouc, The \\ Czech Republic; ${ }^{2}$ Department of \\ Psychology Sciences, Faculty of Social \\ Science and Health Care, Constantine \\ the Philosopher University in Nitra, \\ Nitra, The Slovak Republic; ${ }^{3}$ Institute for \\ Postgraduate Education in Health Care, \\ Prague, The Czech Republic; \\ ${ }^{4}$ Department of Psychiatry, Hospital \\ Liberec, Prague, The Czech Republic
}

Correspondence: Jan Prasko Department of Psychiatry, Faculty of Medicine and Dentistry, University Hospital Olomouc, Olomouc, The Czech Republic

Tel +4206034l4930

Email praskojan@seznam.cz
Purpose: Obsessive compulsive disorder (OCD) is a debilitating mental disorder that often takes a chronic course. One of the factors influencing the treatment effectiveness in anxiety and depressive disorders is the self-stigma. This study focused on the relationship between the self-stigma, symptomatology, and therapeutic outcomes in patients with OCD.

Patients and Methods: Ninety-four inpatients with OCD, who did not sufficiently respond to at least one selective serotonin reuptake inhibitor trial, participated in the study. They attended a six-week therapeutic program consisting of exposure and response prevention, transdiagnostic group cognitive behavioral therapy, individual sessions, mental imagery, relaxation, sport, and ergotherapy. The participants completed several scales: the Internalized Stigma of Mental Illness Scale (ISMI), the self-report Yale-Brown Obsessive Compulsive Scale (Y-BOCS-SR), Beck Anxiety Scale (BAI), Beck Depression Scale-II (BDI-II), and Dissociative Experiences Scale (DES). A senior psychiatrist filled in the Clinical Global Impression (CGI-S).

Results: The average scales' scores considerably declined in all measurements except for DES. The self-stigma positively correlated with all psychopathology scales. It was also higher in patients with a comorbid personality disorder (PD). The higher self-stigma predicted a lower change in compulsion, anxiety, and depressive symptoms but not the change of obsessions or the overall psychopathology.

Conclusion: The self-stigma presents an important factor connected to higher severity of OCD. It is also a minor predictor of a lower change in symptomatology after combined treatment.

Keywords: obsessive compulsive disorder, self-stigma, exposure and response prevention, cognitive behavioral therapy, non-response

\section{Introduction}

Obsessive compulsive disorder (OCD) is a common mental disorder with a debilitating impact on an individual's functioning and quality of life. ${ }^{1}$ Obsession are recurrent and persistent thoughts, images, urges, and impulses that individuals perceive as intrusive and unwanted. ${ }^{2}$ Patients with OCD often feel shameful and guilty for having them. ${ }^{3,4}$ Compulsions are then repetitive actions performed in response to obsessions which can be behavioral as well as cognitive. ${ }^{5}$ Especially when performing noticeable compulsions in public, patients with good insight may feel shame for their actions and fear being labelled as "crazy". 3 
OCD often takes a chronic course, as it takes 10.5 years on average for the individuals to enter the treatment. ${ }^{6}$ One of the significant reasons for this delay is an embarrassment that comes with the symptoms and a fear of being judged and stigmatized. ${ }^{7}$ If a patient enters the treatment, they have a reasonable chance of getting better. Up to $70 \%$ of patients treated with pharmacotherapy experience significant symptom relief, though only 25 to $47 \%$ reach remission. ${ }^{8,9}$ The psychotherapeutic method of choice is cognitive behavioural therapy (CBT), specifically exposure and response prevention (ERP; Öst et $\mathrm{al}^{10}$ ), leading to remission in $52 \%$ of patients. ${ }^{8}$ A combination of CBT and medication may be a viable option in patients with severe OCD or those with comorbidities requiring pharmacotherapy. ${ }^{11}$ A combination of CBT and antidepressant medication (mostly clomipramine or fluvoxamine) may lead to significant clinical improvement in OCD symptomatology. ${ }^{8}$ Still, a considerable number of patients do not sufficiently profit from the current treatment options.

Several main factors influence the treatment outcome. Clinical characteristics, such as lower initial severity of OCD, depressive, ${ }^{12,13}$ and dissociation symptoms, ${ }^{14}$ shorter duration of the disorder, ${ }^{12,13}$ or absence of a personality disorder ${ }^{12}$ predict a better response to $\mathrm{CBT}$ in patients with OCD. Higher severity of anxiety seems to predict worse treatment outcomes. ${ }^{15}$

Another factor that could play a significant role in treatment outcomes is self-stigma. The self-stigma represents a process in which an individual, who gains a socially stigmatized characteristic (such as a mental disorder, Goffman ${ }^{16}$ ), becomes aware of societal prejudices about their condition, agrees with them, and applies them on themselves. ${ }^{17}$ In reaction, they feel increasingly demoralized, hopeless, and helpless. ${ }^{17,18}$ The self-stigma has been related to more severe symptoms of anxiety, depression, and dissociation in patients with depressive and anxiety disorders ${ }^{19}$ and more severe OCD symptoms. ${ }^{20}$ Patients with a personality disorder ${ }^{21,22}$ show higher tendencies towards self-stigmatization, though not all studies find such connection. ${ }^{23}$ In a study by Deres et $\mathrm{al}^{24}$ inpatients with higher self-stigma showed lower engagement and working alliance in psychotherapy. The self-stigma also predicted poorer treatment outcomes in patients with anxiety disorders ${ }^{25}$ and in patients with unipolar depression who underwent a combined treatment. ${ }^{19}$ The selfstigma was identified as a predictor of lower medication adherence in patients with OCD. ${ }^{20}$
Individuals with OCD may experience self-stigma. ${ }^{26}$ Obsessive-compulsive symptoms that contain taboo topics (such as child sexual abuse) ${ }^{27}$ or those that seem bizarre ${ }^{28}$ are more stigmatized by general population than other OCD contents. Patients with these symptoms experience more shame-related barriers to treatment than participants with other types of obsessions. ${ }^{27}$ Thus, shame and stigma present significant obstacles in help-seeking.

To date, the relationship between the self-stigma and the treatment outcomes in individuals with OCD has not been studied. The goal of this study was to explore this topic.

\section{Hypotheses}

Two sets of hypotheses were formulated. It was hypothesized that inpatients with OCD non-responsive to SSRI experience higher self-stigma if they have (a) more intense initial obsessions and (b) compulsions, (c) more severe disorder evaluated by a senior psychiatrist, (d) more intense symptoms of anxiety, (e) depressive symptoms, and (f) dissociation, and ( $\mathrm{g}$ ) if they have a comorbid personality disorder. It was also hypothesized that higher initial self-stigma predicts a lower relative change of (a) obsessions, (b) compulsions, (c) overall mental state evaluated by a senior psychiatrist, (d) anxiety symptoms, and (e) depressive symptoms after combined treatment for OCD.

\section{Methods}

\section{Sample}

The sample consisted of patients with OCD who were hospitalized in a psychotherapeutic ward of a psychiatric department between January 2013 and December 2019. The patient's outpatient psychiatrist first performed psychiatric diagnostics. All patients were evaluated upon admission by a psychiatrist working with the patient during the inpatient care. On the third day of the hospitalization, a senior psychiatrist leading the department also independently evaluated the patient according to the ICD-10 criteria. ${ }^{29}$ During the first day of the hospitalization, the patients completed a set of measurements, including Y-BOCS-SR. The participation was offered during the third day to all patients with OCD who entered the treatment in the mentioned period, did not meet any of the exclusion criteria, and reached Y-BOCSSR score of 16 or higher.

The inclusion criteria presented: the adult age $(18+$ years), OCD diagnosis according to the ICD- $10,{ }^{29}$ a total Y-BOCS-SR score $\geq 16$, completion of measurements, and 


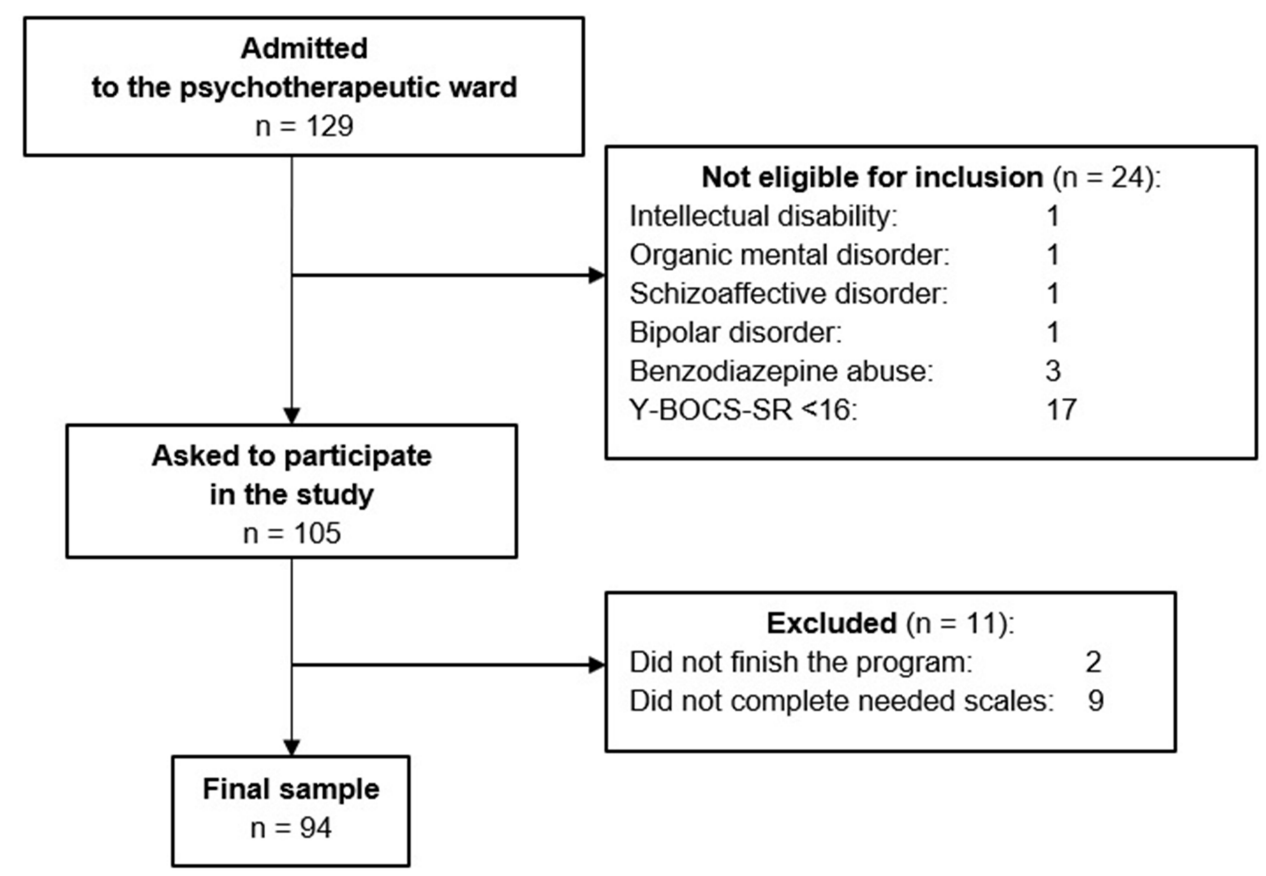

Figure I Flowchart of the enrollment process.

non-response to previous selective serotonin reuptake inhibitor (SSRI) treatment, defined as a total Y-BOCS change $<25 \%$ after at least one SSRI trial lasting three or more months. ${ }^{11}$ The exclusion criteria were: a lifetime diagnosis of organic mental illness, intellectual disability, or psychotic disorder, or a current diagnosis of antisocial personality disorder, bipolar disorder, eating disorder, major depressive disorder, substance abuse disorder, severe physical illness (for example, oncological or severe neurological illness), and current severe suicidal tendencies.

The initial sample consisted of 129 patients. Thirty-five individuals $(27.1 \%)$ could not be included for various reasons (Figure 1). The final sample had 94 participants.

The final sample mostly consisted of women and youngto-middle aged adults who were single and (self-)employed. Most patients were diagnosed with mixed obsessional thoughts and acts (83.0\%). Characteristics are fully described in Table 1 . Since there were only two dropouts, a comparison with completers was not performed.

The most common obsessions were those concerned with contamination $(66.0 \%)$ and aggression $(57.4 \%)$. The most common compulsions presented cleaning or washing (74.5\%), checking (71.3\%), and repeating (54.3\%). On average, the patients declared having $2.32 \pm 1.98$ obsessions and $2.33 \pm 1.91$ compulsions from the Y-BOCS Checklist. Almost half of the patients showed at least a partial improvement at the end of the hospitalization, while 55.3\% remained non-responsive (defined as Y-BOCS-SR change $<25 \%$, Pallanti et $a^{11}{ }^{11}$ ).

The average severity of the OCD symptomatology declined from borderline severe at the start to moderate at the end of the hospitalization (interpretation according to Storch et $\mathrm{al}^{30}$ ). The effect sizes were large, as was the change in the overall severity of the disorder evaluated by a psychiatrist which shifted from "markedly ill" to "mildly ill" on average. The mean level of anxiety improved with a small effect size and was still moderately strong (interpretation according to Julian ${ }^{31}$ ). The change in depressive symptoms was medium. The decline of the dissociative symptoms was negligible. The level of self-stigma, measured at the start of the treatment, was average (T-score: 52 ; based on norms from Ociskova et $\mathrm{al}^{32}$ ) (Table 2). The measurements are described in detail further in the text.

\section{Measures}

Apart from the demographic questionnaire, the participants filled in several questionnaires and scales, some of them repeatedly.

Internalized Stigma of Mental Illness Scale (ISMI; Ritsher et al) consists of 29 items that assess the level of internalized stigma (or self-stigma). ${ }^{33}$ The items form five subscales - alienation, stereotype endorsement, discrimination experience, social withdrawal, and stigma resistance. The patient chooses a number from a 4-point Likert scale 
Table I Demographic and Clinical Characteristics of the Sample

\begin{tabular}{|c|c|c|}
\hline Characteristics & Sub-Characteristics & Count \\
\hline \multicolumn{2}{|l|}{ Number of Patients } & 94 \\
\hline Sex & Male: Female & $41: 53$ \\
\hline Age & Mean in years + Standard deviation & $33.40 \pm 9.72$ \\
\hline Education & Primary: Vocational training: Secondary: Tertiary & 6: 15: $46: 27$ \\
\hline Employment & Student: (Self-)Employed: Unemployed: Disability Rent: Old age pension & 14: 44: 18: 16: 2 \\
\hline Marital Status & Single: Married: Divorced: Widowed & 66: 18: 8: 2 \\
\hline Having a Romantic Partner & Yes: No: Not disclosed & 50: 43: I \\
\hline Positive Family History & None: Different disorder: Same disorder: Not disclosed & 38: 29: 26: I \\
\hline Onset & Age in years + Standard deviation & $19.30 \pm 8.79$ \\
\hline Duration of the Disorder & Mean in years + Standard deviation & $|4.1| \pm 7.92$ \\
\hline Psychiatric Hospitalizations & Number + Standard deviation & $2.80 \pm 4.90$ \\
\hline \multirow[t]{5}{*}{ OCD Diagnosis - Types (ICD-10) } & Yes: No & 94: 0 \\
\hline & F42.0 & 10 \\
\hline & F42.I & 5 \\
\hline & F42.2 & 78 \\
\hline & F42.9 & 1 \\
\hline \multirow[t]{5}{*}{ Comorbid Anxiety Disorder - Types (ICD-I0) } & Yes: No & 23: 71 \\
\hline & F40.0 & 1 \\
\hline & F40.I & 14 \\
\hline & F4I.0 & 5 \\
\hline & F4I.I & 3 \\
\hline \multirow[t]{8}{*}{ Comorbid Personality Disorder - Types (ICD-I0) } & Yes: No & 53: 41 \\
\hline & F60.I & 2 \\
\hline & F60.3 & 31 \\
\hline & F60.4 & 3 \\
\hline & F60.5 & 13 \\
\hline & F60.6 & 1 \\
\hline & F60.7 & 2 \\
\hline & F60.8 (Passive-aggressive) & 1 \\
\hline
\end{tabular}

from 1 (strongly agree) to 4 (strongly disagree). ${ }^{33}$ The total score ranges from 29 to 116 points, with higher scores denoting more severe self-stigma. ${ }^{33}$ The scale shows excellent internal consistency (the Cronbach's $\alpha=0.91$ ), adequate stability in time $(r=0.90)$, and convergent validity (comparisons with the Rosenberg Self-Esteem Scale or the Perceived Devaluation-Discrimination Scale). ${ }^{32}$ This scale's $\alpha$ was 0.89 .

Clinical Global Impression (CGI-S; Guy) is a one-item scale evaluating the overall severity of the mental disorder. ${ }^{34}$ It has two versions - patient and clinician, out of which the latter was used in this study, completed by a senior psychiatrist. The scale consists of seven points describing possible severity of the disorder ranging from 1 (normal - not at all ill) to 7 (among the most extremely ill patients). ${ }^{34}$ The lowest total score is then 1 , the highest value is $7 .^{34}$ The inventory shows excellent stability in time ( $r=0.72-0.82)$ and convergent validity (comparisons with the Hamilton Rating Scale for Depression, the Liebowitz Social Anxiety Scale, or the Disability Profile). ${ }^{35}$

Yale-Brown Obsessive Compulsive Scale - SelfReport (Y-BOCS-SR; Goodman et al, Baer) measures the severity of OCD symptoms during the previous week. ${ }^{36,37}$ It consists of ten items - five for obsessions, five for compulsions. These items evaluate time spent on these symptoms, their interference, caused distress, resistance to them, and control over them. The scale is usually accompanied by a checklist of common types of obsessions and compulsions (for example, contamination obsessions or checking compulsions). Each item is scored on a 5-point Likert scale ranging from 0 ( 0 hours a day spent on obsessions or compulsions, no interference or distress 
Table 2 Mean (SD) and Comparison of Start and End Time Periods of Treatment Scores

\begin{tabular}{|l|l|l|l|}
\hline Variables & Start of the Treatment M(SD) & End of the Treatment M(SD) & Comparison \\
\hline Total obsessive-compulsive symptoms & $26.30 \pm 5.54$ & $21.24 \pm 7.11$ & $\begin{array}{l}t(93)=9.43, p<0.001, \\
\text { Cohen's } d_{z}=0.98\end{array}$ \\
\hline Obsessions & $13.28 \pm 3.03$ & $10.72 \pm 3.70$ & $\begin{array}{l}t(93)=8.71, p<0.001, \\
\text { Cohen's } d_{z}=0.92\end{array}$ \\
\hline Compulsions & $13.02 \pm 3.22$ & $10.49 \pm 3.83$ & $\begin{array}{l}t(93)=7.92, p<0.001, \\
\text { Cohen's } d_{z}=0.8 I\end{array}$ \\
\hline Overall severity of the disorder & $5.23 \pm 0.63$ & $3.10 \pm 0.92$ & $\begin{array}{l}Z=-8.38, p<0.001, \text { Cohen's } \\
d_{z}=2.13\end{array}$ \\
\hline Anxiety & $22.56 \pm 12.42$ & $18.21 \pm 11.75$ & $\begin{array}{l}Z=-3.85, p<0.00 I, C o h e n ' s \\
d_{z}=0.49\end{array}$ \\
\hline Depressive symptoms & $27.02 \pm 12.49$ & $21.78 \pm 14.92$ & $\begin{array}{l}Z=-4.63, p<0.00 I, \text { Cohen's } \\
d_{z}=0.59\end{array}$ \\
\hline Dissociative symptoms & $13.37 \pm 14.15$ & $13.28 \pm 16.25$ & $\begin{array}{l}Z=-1.46, p=0.144, \text { Cohen's } \\
d_{z}=0.02\end{array}$ \\
\hline Self-Stigma & $67.36 \pm 13.82$ & - & - \\
\hline
\end{tabular}

Abbreviations: Cohen's $d_{z}$, the effect size of the standardized mean difference for within subjects design; $t$, the difference between paired observations compared to the null hypothesis value, here an outcome of the dependent $t$-test; $Z$, the rank mean of the group compared to the rank mean of the other group, an outcome of the Wilcoxon signed ranks test; dashes, the self-stigma was measured only at the start of the treatment.

from either of the symptoms, always resists and has control of them) to 4 ( $8+$ hours a day spend on obsession or compulsions, incapacitating symptoms, disabling stress, no resistance or control over the symptoms). The total score ranges from 0 to 40 , separate scores for obsessions and compulsions vary between 0 and $20 .^{36}$ This study used the subjective version of Y-BOCS, which was developed by Baer. ${ }^{37}$ The values of the Cronbach's $\alpha$ ranged from 0.78 to 0.89 . Temporal stability is also good $(r=0.88)$ as is convergent validity evaluated by comparing the self-report version with the clinician ratings. ${ }^{38}$ The alphas were 0.81 for the whole scale, 0.69 for the obsession subscale, and 0.72 for the compulsion subscale in this study.

Beck Anxiety Inventory (BAI; Beck et al) measures the severity of 21 anxiety symptoms on a 4-point Likert scale ranging from 0 (not at all) to 3 (severely). ${ }^{39}$ The patient evaluates their state during the last week. The total score lies between 0 and 63 points. ${ }^{39}$ A score of 17 has been found best to distinguish between healthy controls and patient samples. ${ }^{40}$ The scale shows excellent internal consistency (the Cronbach's $\alpha=0.92$ ), good stability in time $(r=0.75-0.90),{ }^{39,40}$ and moderate validity when compared with the Hamilton Anxiety Rating Scale. ${ }^{40}$ The Cronbach's $\alpha$ was 0.82 in this study.
Beck Depression Inventory - Second edition (BDI-II; Beck et al) measures the severity of 21 depression symptoms during the last two weeks. ${ }^{41}$ Each item has several levels of intensity, and the participants choose the most appropriate for their case. ${ }^{41}$ The lowest item score is 0 (for example, Sadness: I do not feel sad.), highest item score is 3 (Sadness: I am so sad or unhappy that I cannot stand it.). The total score lies between 0 and 63 points. $^{41}$ A cut-off score of 17 best differentiates between non-clinical samples and depressed individuals. ${ }^{42}$ The internal consistency of the scale is excellent (the Cronbach's $\alpha=0.90$ ), stability in time is good $(r=0.77-0.83),{ }^{42}$ as is the convergent validity (comparisons with CGI and the diagnosis of an affective disorder) and discriminative validity (a comparison with the Adult Dispositional Hope Scale and the Satisfaction with Life Scale). ${ }^{41,42}$ The $\alpha$ was 0.92 in this study.

Dissociation Experience Scale (DES; Carlson \& Putnam) contains 28 items focusing on dissociative experiences that range from depersonalization and derealization to dissociative amnesia and discontinuation of awareness. ${ }^{43}$ The patients mark a spot on a $10-\mathrm{cm}$ line according to how much time per day they usually experience dissociative symptoms. The lowest item score is 0 
(never); the highest item score is 100 (always). The total score lies between 0 and $100 .^{43}$ The internal consistency is excellent (the Cronbach's $\alpha=0.88-0.92$ ), temporal stability is good ( $r=0.81-0.84),{ }^{44}$ and the validity (comparisons with the Tellegen Absorption Scale, the Ambiguity Intolerance Scale, or the diagnosis of a dissociative disorder). ${ }^{43}$ The Cronbach's $\alpha$ was 0.96 in the presented study.

\section{Primary and Secondary Outcome Measures}

The main indicators of the treatment effect were the relative change of the obsessive-compulsive symptomatology and the overall psychopathology. Secondary outcome measures were the relative changes in anxiety, depressive symptoms, and dissociation.

\section{Methods of Treatment Medication}

Patients were mostly treated with psychopharmacs prescribed by their outpatient psychiatrist. A minority was currently medication-free $(n=8)$. The mean dosages of medication were minimally changed, only when warranted by patients' needs (mostly gradual discontinuation of anxiolytics). The average antidepressant dosage did not significantly change during the treatment (the starting dosage was $47.72 \pm 24.78 \mathrm{mg}$ of paroxetine equivalent $(n=83)$, the ending dosage was $46.86 \pm 24.95 \mathrm{mg}(n=$ 85); Wilcoxon signed ranks test (WSRT): $Z=-0.06, p=$ 0.955). The same was true of the average dosages of anxiolytics (the starting dosage was $11.30 \pm 6.94 \mathrm{mg}$ of diazepam equivalent $(n=23)$, the ending dosage was $13.18 \pm 9.82 \mathrm{mg}(n=11)$; WSRT: $Z=-1.00, p=0.317)$ and antipsychotics (the starting dosage was $1.61 \pm 1.68 \mathrm{mg}$ of risperidone equivalent $(n=32)$, the ending dosage was $1.28 \pm 1.08 \mathrm{mg}(n=35)$; WSRT: $Z=-1.12, p=0.263)$. Ten patients were using mood stabilizers at the start of the hospitalization (the starting dosage was $248.00 \pm 89.67 \mathrm{mg}$ of lamotrigine equivalent), and the number decreased to eight patients in the end (the dosage was $243.75 \pm$ $102.81 \mathrm{mg})$. Mood stabilizers were mostly administered to patients with a comorbid borderline personality disorder.

\section{Psychotherapy}

The psychotherapeutic program lasted six weeks. The main parts were daily exposure with response prevention, transdiagnostic group cognitive behavioral therapy (CBT; 90-minutes session per day, 20 sessions per program), and one 90-minutes community session per day (totally 25 sessions) that aimed at exposures, skills training, and development of interpersonal relationships. The CBT groups focused on psychoeducation, case conceptualization with a list of symptoms and life problems, cognitive restructuring and schema work, problem-solving, and exposures with response prevention (these were performed both during and outside of the sessions). ${ }^{45}$ The program was supplemented with a weekly session of mental imagery and daily relaxation, sport, and ergotherapy. Each patient also underwent five individual sessions with the CBT therapist. These sessions served for goal planning for the next week (especially exposures). The individuals also could discuss sensitive topics with the therapist that they felt were too intimate for presentation in the group. When needed, these individual sessions focused on therapeutic work with trauma, such as imagery rescripting.

\section{Statistics}

Statistical programs SPSS 24.0 and G*Power 3.1.9.2 were used for the calculations. ${ }^{46}$ Apart from descriptive statistics and sample and effect size calculations, the applied statistics included comparative (chi-square, dependent, and independent t-tests or their non-parametric equivalents) and inferential tests (Pearson's and Spearman correlations and multiple linear regression). The Shapiro-Wilk test assessed the normality of the data. Sample size estimation concerning the first set of hypotheses was based on data from the studies of Ansari et al $(N=112, r=0.61$ for the connection between the self-stigma and obsessivecompulsive symptoms), ${ }^{20}$ Ociskova et al $(N=109, r=$ 0.31 for the self-stigma and overall severity of the mental disorder, $r=0.33$ for the self-stigma and anxiety, $r=0.55$ for the self-stigma and depressive symptoms, and $r=0.44$ for the self-stigma and dissociation), ${ }^{22}$ and Grambal et al ( $N=184$, Cohen's $d=0.52$ for the self-stigma in patients with and without a personality disorder $)^{21}$ with Prasko et al ( $N=63$, Cohen's $d=0.60$ for the same purpose). ${ }^{19}$ The estimation of the second set of hypotheses was based on data from the studies of Prasko et al $(N=63, r=0.50$ for the self-stigma and the relative change of overall severity of the mental disorder and $r=0.27$ for the relative change of the depressive symptoms) ${ }^{19}$ and Ociskova et al ( $N=109, r=0.26$ the self-stigma and the relative change of anxiety). ${ }^{25}$ The effect sizes were interpreted according to Cohen's guideline. ${ }^{47}$ The interpretation of $r$ values followed subsequent cut-off values: negligible: $<0.10$; small: 
Table 3 Correlation Coefficients of the Relationship Between the Self-Stigma and Psychopathology at the Start of the Treatment

\begin{tabular}{|l|l|l|}
\hline Variables & Correlation & $\mathbf{P}$ \\
\hline Total obsessive-compulsive symptoms & $0.4 \mathrm{I}^{\mathrm{P}}$ & $<0.00 \mathrm{I}$ \\
Obsessions & $0.39^{\mathrm{P}}$ & $<0.00 \mathrm{I}$ \\
Compulsions & $0.33^{\mathrm{P}}$ & 0.002 \\
Overall severity of the disorder & $0.28^{\mathrm{S}}$ & 0.008 \\
Anxiety & $0.44^{\mathrm{S}}$ & $<0.00 \mathrm{I}$ \\
Depressive symptoms & $0.68^{\mathrm{P}}$ & $<0.00 \mathrm{I}$ \\
Dissociative symptoms & $0.39^{\mathrm{S}}$ & $<0.00 \mathrm{I}$ \\
\hline
\end{tabular}

Note: The choice was based on the results of the Shapiro-Wilk test. Abbreviation: P or S, Pearson's or Spearman correlation coefficient.

$0.10-0.29$; medium: $0.30-0.49$; and large: $\geq 0.50$. The $d$ values were interpreted as: negligible: $<0.20$; small: 0 .20-0.49; medium: 0.50-0.79; and large: $\geq 0.80$. The $f$ value was interpreted as negligible if $<0.10$; small: $0.10-0.24$; medium: $0.25-0.39$, and large: $\geq 0.40 .^{47}$ The level of statistical significance was set at $\mathrm{p}<0.05$, power at $80 \%$.

\section{Ethics}

The research was performed in agreement with the Helsinki Declaration and the Guideline for Good Clinical Practice. $^{48}$ The ethical committee of the University Hospital Olomouc accepted the design of the study in December 2009. Registration number: NT11001 VES2010. All participants signed informed consent.

\section{Results}

\section{Self-Stigma and Initial Severity of Psychopathology}

The first set of hypotheses focused on the relationship between the self-stigma and chosen psychological factors. It was hypothesized that the inpatients with the higher self-stigma experience more intense obsessions and compulsions, more severe disorder evaluated by a senior psychiatrist, and more intense symptoms of anxiety, depressive symptoms, and dissociation. A correlation analysis was applied to test these predictions. Consistent with the expectations, the higher selfstigma was related to more intense OCD symptoms and more severe overall severity, anxiety, depressive symptoms, and dissociation (Table 3). Most of these connections were moderate in their effect size. The exceptions were a small-sized correlation coefficient between the self-stigma and the overall severity of the mental disorder and a large connection between the self-stigma
Table 4 Correlation Coefficients of the Relationship Between the Self-Stigma and Relative Change of Psychopathology

\begin{tabular}{|l|l|l|}
\hline Variable & Correlation & $\boldsymbol{P}$ \\
\hline Total obsessive-compulsive symptoms & $-.29^{\mathrm{P}}$ & 0.006 \\
Obsessions & $-.20^{\mathrm{P}}$ & 0.065 \\
Compulsions & $-.28^{\mathrm{S}}$ & 0.007 \\
Overall severity of the disorder & $-.13^{\mathrm{P}}$ & 0.229 \\
Anxiety & $-.27^{\mathrm{S}}$ & 0.009 \\
Depressive symptoms & $-.41^{\mathrm{S}}$ & $<0.001$ \\
\hline
\end{tabular}

Note: The choice was based on the results of the Shapiro-Wilk test.

Abbreviation: P or S, Pearson's or Spearman correlation coefficient.

and the depressive symptoms. The last sub-hypothesis assumed more intense self-stigma among individuals with a comorbid personality disorder. This assumption was tested with the independent $t$-test. The result showed that individuals with a comorbid personality disorder stigmatize themselves more than patients without the comorbidity $(70.18 \pm 12.59$ versus $63.90 \pm$ 14.61, respectively; independent $t$-test: $t(87)=-2.179$, $p=0.032$, Cohen's $d: 0.34$ ), though the difference between the groups was small. All sub-hypotheses were supported.

\section{Self-Stigma and Treatment Outcomes in OCD}

The second set of hypotheses explored the relationships between the initial self-stigma and selected outcomes of combined inpatient treatment. It was hypothesized that the higher initial self-stigma predicts a lower relative change of (a) obsessions, (b) compulsions, (c) overall mental state evaluated by a senior psychiatrist, (d) anxiety symptoms, and (e) depressive symptoms. Another correlation analysis was computed to test these predictions (Table 4). The first sub-hypothesis focusing on the relationship between the self-stigma and the relative change in the severity of the obsessions was not supported. Although there was a small negative connection between these two variables, it was only trending towards significance. The sub-hypothesis concerning the relative change of the overall severity of the disorder was also not supported; the connection between the variables was small in size. The rest of the subhypotheses were supported. The relations between the self-stigma and the relative changes of compulsions and anxiety were small in their sizes, while the connection between the former and the relative change of depressive symptoms was moderate. 
Table 5 Multiple Regression Analysis with the Self-Stigma as a Dependent Variable

\begin{tabular}{|l|l|l|l|l|l|}
\hline Variables & $\boldsymbol{B}$ & SE & $\boldsymbol{\beta}$ & $\boldsymbol{t}$ & $\boldsymbol{P}$ \\
\hline Constant & 25.725 & 9.625 & & 2.673 & 0.009 \\
Obsessions & -0.078 & 0.466 & -0.017 & -0.167 & 0.868 \\
Compulsions & 0.538 & 0.410 & 0.124 & 1.312 & 0.193 \\
$\begin{array}{l}\text { Overall severity of } \\
\text { the disorder }\end{array}$ & 2.749 & 1.731 & 0.128 & 1.588 & 0.116 \\
$\begin{array}{l}\text { Anxiety } \\
\text { Depressive } \\
\text { symptoms } \\
\begin{array}{l}\text { Dissociative } \\
\text { symptoms }\end{array}\end{array}$ & 0.119 & 0.112 & 0.110 & 1.069 & 0.288 \\
Personality disorder & 0.567 & 0.118 & 0.514 & 4.801 & 0.001 \\
\hline
\end{tabular}

Notes: Adjusted $R^{2}: 0.491$. ANOVA: $F=13.123 ; d f=7 ; p<0.001$

\section{Factors Predicting Self-Stigma: Post Hoc Analysis}

An additional regression analysis was performed to place the results in a broader context. It focused on the identification of the strongest factors connected with the selfstigma. The specific method was Enter. The independent variables were those factors that showed at least a small connection with the self-stigma in the correlation analysis the severity of the obsessions and compulsions and overall psychopathology, depressive, anxiety, and dissociative symptoms, and the presence of a comorbid personality disorder. All factors were initially checked for collinearity. The resultant model had a large effect size (Cohen's $f^{2}=$ 0.96). Most relations between the self-stigma and other variables showed negligible effect sizes (evaluated by the beta values). The exceptions were the relation with the depressive symptoms with large effect size and the presence of a comorbid personality disorder with a small to negligible effect (Table 5).

\section{Discussion}

This study had two goals - to explore the relationship between the self-stigma and severity of psychopathology in patients with OCD and to explore the connection between the self-stigma and the outcomes of combined psychotherapy and pharmacotherapy. The sample consisted of patients with OCD who were admitted to an inpatient psychotherapeutic program and who had not benefited sufficiently from at least one SSRI trial. The criterion presented the first level of non-response and was chosen because all other levels include non-response to CBT which is mostly inaccessible in the Czech Republic. ${ }^{11}$
A total of 129 patients with OCD were admitted to the psychotherapeutic ward, out of which 94 presented the final sample. The most common reasons for the noninclusion were low severity of OCD and insufficient completion of the scales. There were more women than men in the sample (56.4\%), but the proportion was close to the $1: 1$ gender ratio observed in this population. ${ }^{49}$ The group consisted of young to middle-aged adults. The comorbidity rates of the anxiety disorders were similar to those found by Brakoulias et al. ${ }^{50}$ In contrast, the anankastic personality disorder was slightly under-represented (compared with the Brakoulias et al sample) and the borderline personality disorder was over-represented (compared with Melca et $\mathrm{al}^{51}$ ). Most patients were treated with psychopharmaceuticals prescribed by their outpatient psychiatrist; the changes in medication during the hospitalization were negligible.

The overall severity of the OCD symptomatology mainly decreased during the treatment. Still, the extent of this decrease was lower than shown by other studies using the group therapy (pre-post effect size (ES): 0.98 in this study, 1.18 in the meta-analysis of Jónsson \& Hougaard $^{52}$ ) which might have been due to the differences between inpatient and outpatient programs. While the inpatient programs are more intensive, the outpatient groups bring a benefit of having more time to practice between the sessions, which may be crucial for the improvement. ${ }^{53}$ The change of the anxiety was small in size, and the improvement of the depressive symptoms was moderate. Both results are comparable to the extent found in patients with depressive or neurotic spectrum disorders undergoing the combined treatment. ${ }^{19,25}$ The change of the dissociative symptoms was negligible. This result is in line with findings of Prasko et $\mathrm{al}^{54}$ who reported a minimum change in dissociative symptoms (DES score at the start and the end of the treatment: $14.30 \pm 15.39$ versus $13.98 \pm 16.03$ points) in patients with neurotic spectrum disorders or major depression who underwent a six-week combined inpatient program. Individuals with OCD frequently struggle with dissociation, ${ }^{55}$ which was linked to lower treatment response. ${ }^{14}$ As Belli mentions, the standard cognitive behavioral treatment of patients with OCD may need to be supplemented with additional approaches to decrease dissociation, namely therapeutic work aiming at experienced childhood adversities and their consequences. ${ }^{56}$

The level of the self-stigma was average; the mean item value of the ISMI scale was comparable to a value 
reported by Picco et al. ${ }^{26}$ All hypotheses focusing on the connections between the self-stigma and initial levels of psychopathology were supported. The relationship between the self-stigma and severity of the OCD symptoms was medium in size, which differs from Ansari et al, who found a large connection $(r=0.61$ for the total score of Y-BOCS). ${ }^{20}$ The difference in the effect sizes may be potentially explained by higher levels of the self-stigma and the presence of comorbid major depression $(23.2 \%$ of the participants) in the Ansari et al sample. The current study sample experienced more severe OCD (the total Y-BOCS-SR score was $26.30 \pm 5.54$ versus $20.37 \pm$ $8.31)$ but relatively lower self-stigma. The correlation between the OCD symptoms and the self-stigma thus reached a smaller effect size than in the sample of Ansari et al.

The connection between the self-stigma and the overall severity of psychopathology, assessed by a psychiatrist, showed a small effect size. In a study by Ociskova et al, who focused on patients with anxiety disorders, this correlation was similarly small $(r=0.31) .{ }^{25}$ In both studies, the Clinical Global Impression was the only outcome measurement that was not self-reported. The evaluation of the psychiatrist provides "an outside perspective" on the patients' struggles. However, this external assessment sometimes cannot sufficiently grasp the subjective experience of the patients. Some patients feel ashamed of their symptoms, especially when they contain taboo topics. ${ }^{27}$ They may not share the whole extent of their symptoms with the psychiatrist who then scores their disorder as less severe than it really is. However, the self-stigma measurement is self-reported. ${ }^{33}$ The patient may freely report the extent of their feelings of shame or self-doubt. If the psychiatrist does not know all information necessary for a valid assessment, the disconnect between the selfevaluative measure and the clinician-based measure may result in a smaller effect size than expected.

The self-stigma moderately correlated with dissociation in an extent observed in patients with anxiety disorders $(r=0.39$ in the presented sample versus 0.44 in the sample of Ociskova et $\mathrm{al}^{22}$ ). Both effect sizes were medium. This shows that the concepts are related but still independent. Ociskova et al found that dissociation may present a predictor of the self-stigma and that the harm avoidance mediates this influence. ${ }^{22}$ This finding needs to be replicated by other studies. If it is successfully replicated, it would suggest that the self-stigma can result from early adverse experiences that reinforced dissociation as a coping mechanism and contributed to self-esteem issues that tightly connect with the self-stigma. ${ }^{18}$

The connection of the self-stigma and with the anxiety was moderate in size and similarly robust as in the case of Ociskova et al ( $r=0.44$ versus 0.33 ). ${ }^{22}$ This comparison shows a small variance in the strength of the connection, which may have been caused by different samples (OCD patients versus individuals with various anxiety disorders). ${ }^{22}$ However, both effect sizes were medium. This suggests that this connection may be transdiagnostic. The causality of this relationship is unclear because the studies have been cross-sectional. The higher self-stigma may result in more pronounced anxiety over time due to the fear that one could be identified as "mentally ill". On the other side, higher anxiety sensitivity leads an individual to apprehend the anxiety symptoms - physical symptoms, a possibility of losing control and social rejection. ${ }^{57}$ Since anxiety sensitivity also occurs in individuals with OCD ${ }^{58}$ the self-stigma can potentially present a consequence rather than a cause of exacerbated anxiety. However, the relationship between anxiety sensitivity and the self-stigma has not been studied yet.

Reaching a large effect size, the stigma showed a more robust connection with the depressive symptoms than Ociskova et al ( $r=0.68$ versus 0.55$){ }^{22}$ The levels of the self-stigma can explain this and the depressive symptoms, both of which were higher and had bigger standard deviations in the presented sample than in the sample of Ociskova et al. The result may have also been influenced by a difference in the homogeneity of the samples (for example, almost a third of the sample of Ociskova et $\mathrm{al}^{22}$ had a comorbid depression). Corrigan et $\mathrm{al}^{17}$ found that the self-stigma precedes exacerbation of depressive symptoms. It often leads to a loss of self-esteem and the "Why Try" phenomenon. ${ }^{17}$ When a person applies the negative stereotypes on themselves, they can start feeling more unworthy and incapable, which then results in more severe depressive symptoms. ${ }^{17}$ The self-stigma may then present a cause of the exacerbation of the depressive symptoms.

The self-stigma was also higher in the subgroup with a comorbid personality disorder. The difference between the participants with and without this comorbidity was fairly small (Cohen's $d$ : 0.34) compared with that reported by Grambal et $\mathrm{al}^{21}$ and Prasko et $\mathrm{al}^{19}$ (0.52 and 0.60), respectively. This difference may be caused by variability among the samples. Prasko et $\mathrm{al}^{19}$ studied individuals with major depression, some of whom with a comorbid 
personality disorder (comorbid $n=17$, non-comorbid $n=$ 46), while Grambal et $\mathrm{al}^{21}$ had a heterogenous sample of individuals with schizophrenia, bipolar disorder, anxiety disorders, major depression, or a borderline personality disorder (personality disorder $n=35$, others $n=149$ ). Prasko et al did not describe the specific types of personality disorders present in the sample. It is possible that their sample included more comorbid patients who were prone towards self-stigmatization than ours (such as individuals with a borderline personality disorder ${ }^{21}$ ). Grambal et $\mathrm{al}^{21}$ focused solely on the borderline personality disorder in their analysis. In the current study, 53 patients had a comorbid personality disorder out of which 31 had the borderline subtype. Patients with a borderline personality disorder may be especially vulnerable towards selfstigmatization. $^{21}$ The self-stigma may reflect self-concept issues that are prevalent in this population as well as their sensitivity towards rejection. ${ }^{26}$ These individuals also show higher tendencies towards feeling shame which negatively affects their self-esteem. ${ }^{59}$ A subsample consisting solely of participants with the borderline personality disorder may then reach higher scores in the selfstigma measures than a subsample of individuals with various personality disorders whose vulnerability towards self-stigmatization may be lower. Since there has been a lack of studies on personality disorders and self-stigma, this issue presents an important topic for future research.

The next part of the study focused on the relationship between the self-stigma and the treatment outcomes. The second set of hypotheses expected the self-stigma to decrease the treatment effectiveness evaluated by relative changes of obsessions, compulsions, overall severity of the disorder, anxiety, and depressive symptoms. The first subhypothesis focusing on the relative change of obsessions was not supported. There was a small negative connection between the self-stigma and the relative change of obsessions, but it was only trending towards significance. Although a bigger sample size would yield a significant result, the effect size would likely remain small. ${ }^{60}$ The presented study has been the first to explore this topic. Looking at findings of Prasko et $\mathrm{al}^{19}$ and Ociskova et $\mathrm{al}^{24}$ who focused on the self-stigma and relative changes of depressive symptoms $(r=0.27)^{19}$ and anxiety symptoms $(r=0.26),{ }^{24}$ it seems that the self-stigma is a minor factor influencing the treatment outcomes - when it comes to obsessions or other symptoms. The sub-hypothesis focusing on the relative change of compulsions was supported.
However, the effect size of this connection was also small. Smaller change of the OCD symptoms could be potentially explained by lower engagement, and worse working alliance in psychotherapy observed among inpatients with a higher self-stigma. ${ }^{24}$ The treatment of OCD requires highly active cooperation and sustained willingness to change on the part of the patient. The person needs to perform daily exposures and work actively and systematically to resolve their symptoms. ${ }^{10}$ Self-stigmatizing patients, who are demoralized, may struggle with performing needed therapeutic activities which would then prevent sufficient improvement.

The sub-hypothesis on the self-stigma and the overall change of psychopathology was not supported. The effect size of this connection was small and non-significant. This finding is not in line with the results of Ociskova et $\mathrm{al}^{23}$ $(r=0.28)$ and Prasko et $\mathrm{al}^{19}(r=0.50)$ who studied patients with anxiety and depressive disorders. Differences can explain this among the samples. Future studies may benefit from focusing on the identification of specific criteria that psychiatrists follow while scoring CGI. Another option would be using a more detailed version of CGI, such as CGI for borderline personality disorder (when studying this population). ${ }^{61}$

The sub-hypothesis expecting the lower change of anxiety symptoms with the higher self-stigma was supported. We observed a small effect size which is consistent with the result of Ociskova et al $(r=0.26$ versus $r=-0.27$ in the current study). ${ }^{25}$ The self-stigma seems to maintain the anxiety during the treatment and thus prevent the anxious symptomatology from decreasing. However, the influence of self-stigma is the only minor. The last sub-hypothesis focused on the relative change of depressive symptoms. With a moderate effect size, this sub-hypothesis was supported. The effect size was larger than reported by Prasko et al in a sample of depressed inpatients $(r=0.27$ versus $r=-0.41) .{ }^{19}$ The difference may be caused by variations between the diagnostic groups and the applied therapeutic strategies. The exposure and response therapy (ERP) requires the patient to be rather courageous and active in their treatment. $^{10}$ The demoralizing influence of the selfstigma may then influence the patients with OCD more than others. Since the self-stigma exacerbates the feelings of helplessness and hopelessness and beliefs of incapability, ${ }^{17,18}$ it may impact demanding treatments such as ERP more. 


\section{Limitations}

The study has several significant limitations. First, it was performed in a natural setting which led to a heterogeneous sample, and certain variability in the treatment - some patients with OCD had more copatients with the same disorder in the group than others, several therapists provided the treatment during the years, the patients were using various medication. A homogenous group could have enhanced the therapeutic outcomes and provide more generalizable results. If the patients were divided into groups receiving different types of treatment, we could have studied the relations between the self-stigma and various treatment modalities. Since the patients underwent a combined treatment, this was not possible.

Additionally, the patients could have completed the self-stigma measure at the end of the treatment to show if the self-stigma changes during the treatment. The clinician version of Y-BOCS may have brought more representative results. Moreover, it would have allowed exploring a potential effect of insight (or avoidance) on the self-stigma and the treatment outcomes, as shown in studies of individuals with schizophrenia. ${ }^{62,63}$ The sample size prevented performing several statistical analyses that could have been interesting - namely the difference between the self-stigma in subgroups (patients with and without "taboo" symptoms) and structural equation modeling that could have explored the complex relationship among the self-stigma and the therapeutic outcomes. The structural equation modeling could have trimmed the correlational results to highlight the most critical connections. However, the sample size was too small to allow more indepth analyses.

\section{Future Research}

The future research should focus on mediation and moderation analyses that would identify ways in which the self-stigma decreases the treatment effectiveness. This might be especially useful in studies focusing on one type of treatment. Measuring potential changes of the selfstigma during the treatment may also be useful, as can be an exploration of the potential connection with insight or avoidance. Furthermore, the studies should focus on identifying treatment adjustments that would bring benefit to patients with pronounced depressive symptoms who undergo similarly intensive OCD therapy.

\section{Conclusion}

This paper identified the self-stigma connected to increased severity of OCD symptoms, anxiety, depression, dissociation, and overall symptomatology. The self-stigma was higher in individuals with a comorbid personality disorder. It predicted smaller treatment effectiveness. However, the strongest predictor of a response to the combined treatment was the initial severity of depressive symptoms. Future research should focus on tackling depressive symptoms in time limited therapies and mediation analyses connecting the self-stigma with the decreased therapeutic outcomes.

\section{Funding}

This research did not receive any specific grant from funding agencies in the public, commercial, or not-forprofit sectors.

\section{Disclosure}

The authors declare no conflicts of interest in this work.

\section{References}

1. Huppert JD, Simpson HB, Nissenson KJ, Liebowitz MR, Foa EB. Quality of life and functional impairment in obsessive-compulsive disorder: A comparison of patients with and without comorbidity, patients in remission, and healthy controls. Depress Anxiety. 2009;26 (1):39-45. doi:10.1002/da.20506

2. American Psychiatric Association. Diagnostic and Statistical Manual of Mental Disorders (5th Ed.). Arlington, VA: Author; 2013.

3. Weingarden H, Renshaw KD. Shame in the obsessive compulsive related disorders: a conceptual review. $J$ Affect Disord. 2015;171:74-84. doi:10.1016/j.jad.2014.09.010

4. Basile B, Mancini F, Macaluso E, Caltagirone C, Bozzali M. Abnormal processing of deontological guilt in obsessive-compulsive disorder. Brain Struct Funct. 2014;219(4):1321-1331. doi:10.1007/ s00429-013-0570-2

5. Williams MT, Farris SG, Turkheimer E, et al. Myth of the pure obsessional type in obsessive-compulsive disorder. Depress Anxiety. 2011;28(6):495-500. doi:10.1002/da.20820

6. Prasko J, Raszka M, Adamcova K, et al. Predicting the therapeutic response to cognitive behavioural therapy in patients with pharmacoresistant obsessive-compulsive disorder.. Neuro Endocrinol Lett. 2009;30(5):615-623

7. Torres AR, Prince MJ. A importância de estudos epidemiológicos sobre o transtorno obsessivo-compulsivo. Rev Brasileira De Psiquiatria. 2004;26(3):141-142. doi:10.1590/s151644462004000300001

8. Simpson HB, Huppert JD, Petkova E, Foa EB, Liebowitz MR. Response versus remission in obsessive-compulsive disorder. J Clin Psychiatry. 2006;67(2):269-276. doi:10.4088/jcp.v67n0214

9. Fineberg NA, Gale TM. Evidence-based pharmacotherapy of obsessive-compulsive disorder. Int J Neuropsychopharmacology. 2005;8 (1):107-129. doi:10.1017/S1461145704004675

10. Öst L-G, Havnen A, Hansen B, Kvale G. Cognitive behavioral treatments of obsessive-compulsive disorder. A systematic review and meta-analysis of studies published 1993-2014. Clin Psychol Rev. 2015;40:156-169. doi:10.1016/j.cpr.2015.06.003 
11. Pallanti S, Hollander E, Bienstock C, Koran L, Leckman J, Marazziti D. International Treatment Refractory OCD Consortium. Treatment non-response in OCD: methodological issues and operational definitions. Int $J$ Neuropsychopharmacology. 2002;5 (2):181-191. doi:10.1017/S1461145702002900

12. Eisen JL, Sibrava NJ, Boisseau CL, et al. Five-year course of obsessive-compulsive disorder: predictors of remission and relapse. J Clin Psychiatry. 2013;74(3):233-239. doi:10.4088/JCP.12m07657

13. Jakubovski E, Diniz JB, Valerio C, et al. Clinical predictors of long-term outcome in obsessive-compulsive disorder. Depress Anxiety. 2013;30(8):763-772. doi:10.1002/da.22013

14. Semiz UB, Inanc L, Bezgin CH. Are trauma and dissociation related to treatment resistance in patients with obsessive-compulsive disorder? Soc Psychiatry Psychiatr Epidemiol. 2014;49(8):1287-1296. doi:10.1007/s00127-013-0787-7

15. Knopp J, Knowles S, Bee P, Lovell K, Bower P. A systematic review of predictors and moderators of response to psychological therapies in OCD: do we have enough empirical evidence to target treatment? Clin Psychol Rev. 2013;33(8):1067-1081. doi:10.1016/j.cpr.2013.08.008

16. Goffman E. Stigma: Notes on the Management of Spoiled Identity. New York: Touchstone; 1986.

17. Corrigan PW, Nieweglowski K, Sayer J. Self-stigma and the mediating impact of the "why try" effect on depression. J Community Psychol. 2019;47(3):698-705. doi:10.1002/jcop.22144

18. Livingston JD, Boyd JE. Correlates and consequences of internalized stigma for people living with mental illness: A systematic review and meta-analysis. Soc Sci Med. 2010;71(12):2150-2161. doi:10.1016/j. socscimed.2010.09.030

19. Prasko J, Ociskova M, Grambal A, Sigmundova Z. Slepecky M. Personality features, dissociation, self-stigma, hope, and the complex treatment of depressive disorder. Neuropsychiatric Dis Treat. 2016;12:2539-2552. doi:10.2147/NDT.S117037

20. Ansari E, Mishra S, Tripathi A, Kar SK, Dalal PK. Cross-sectional study of internalised stigma and medication adherence in patients with obsessive compulsive disorder. General Psychiatry. 2020;33 (2):e100180. doi:10.1136/gpsych-2019-100180

21. Grambal A, Prasko J, Kamaradova D, et al. Self-stigma in borderline personality disorder - Cross-sectional comparison with schizophrenia spectrum disorder, major depressive disorder, and anxiety disorders. Neuropsychiatr Dis Treat. 2016;(2016(12):2439-2448. doi:10.2147/ NDT.S114671

22. Ociskova M, Prasko J, Kamaradova D, Grambal A, Sigmundova Z. Individual correlates of self-stigma in patients with anxiety disorders with and without comorbidities. Neuropsych Dis Treat. 2015;11:1767-1779. doi:10.2147/NDT.S87737

23. Ociskova M, Prasko J, Vrbova K, Kasalova P, Holubova M. Selfstigma and treatment effectiveness in patients with anxiety disorders a mediation analysis. Neuropsychiatric Dis Treat. 2018;14:383-392. doi:10.2147/NDT.S152208

24. Deres AT, Bürkner P-C, Klauke B, Buhlmann U. The role of stigma during the course of inpatient psychotherapeutic treatment in a German sample. Clin Psychol Psychother. 2020;27(2):239-248. doi:10.1002/cpp. 2423

25. Prasko J, Ociskova M, Latalova K, Kamaradova D, Grambal A. Psychological factors and treatment effectiveness in resistant anxiety disorders in highly comorbid inpatients. Neuropsychiatric Dis Treat. 2016;12:1539-1551. doi:10.2147/NDT.S104301

26. Picco L, Lau YW, Pang S, et al. Mediating effects of self-stigma on the relationship between perceived stigma and psychosocial outcomes among psychiatric outpatients: findings from a cross-sectional survey in Singapore. BMJ Open. 2017;7(8):e018228. doi:10.1136/bmjopen-2017-018228

27. Glazier K, Wetterneck C, Singh S, Williams M. Stigma and shame as barriers to treatment for obsessive-compulsive and related disorders. $J$ Depression Anxiety. 2015;4(3):191. doi:10.4191/21671044.1000191
28. Stengler-Wenzke K, Trosbach J, Dietrich S, Angermeyer MC. Experience of stigmatization by relatives of patients with obsessive compulsive disorder. Arch Psychiatr Nurs. 2004;18(3):88-96. doi:10.1016/j.apnu.2004.03.004

29. World Health Organization. The ICD-10 Classification of Mental and Behavioural Disorders: Clinical Descriptions and Diagnostic Guidelines. Geneva: Author; 1992.

30. Storch EA, De Nadai AS, Conceição Do Rosário M. Defining clinical severity in adults with obsessive-compulsive disorder. Compr Psychiatry. 2015;63:30-35. doi:10.1016/j.comppsych.2015.08.007

31. Julian LJ. Measures of anxiety: state-Trait Anxiety Inventory (STAI), Beck Anxiety Inventory (BAI), and Hospital Anxiety and Depression Scale-Anxiety (HADS-A). Arthritis Care Res (Hoboken). 2011;63 (S11):S467-S472. doi:10.1002/acr.20561

32. Ociskova M, Prasko J, Kamarádová D, et al. Self-stigma in psychiatric patients--standardization of the ISMI scale.. Neuro Endocrinol Lett. 2014;35(7):624-632.

33. Ritsher JB, Otilingam PG, Grajales M. Internalized stigma of mental illness: psychometric properties of a new measure. Psychiatry Res. 2003;121(1):31-49. doi:10.1016/j.psychres.2003.08.008

34. Guy W. ECDEU Assessment Manual for Psychopharmacology. Rockville: DHEW; 1976.

35. Zaider TI, Heimberg RG, Fresco DM, Schneier FR, Liebowitz MR. Evaluation of the clinical global impression scale among individuals with social anxiety disorder. Psychol Med. 2003;33(4):611-622. doi:10.1017/s0033291703007414

36. Goodman WK. The Yale-Brown Obsessive Compulsive Scale. I. Development, use, and reliability. Arch Gen Psychiatry. 1989;46 (11):1006-1011. doi:10.1001/archpsyc.1989.01810110048007

37. Baer L, Brown-Beasley MW, Sorce J, Henriques AI. Computerassisted telephone administration of a structured interview for obsessive-compulsive disorder. Am $J$ Psychiatry. 1993;150 (11):1737-1738. doi:10.1176/ajp.150.11.1737

38. Rapp AM, Bergman RL, Piacentini J, McGuire J. Evidence-Based Assessment of Obsessive-Compulsive Disorder. J Cent Nerv Syst Dis. 2016;8:13-29. doi:10.4137/JCNSD.S38359

39. Beck AT, Epstein N, Brown G, Steer RA. An inventory for measuring clinical anxiety: psychometric properties.. J Consult Clin Psychol. 1988;56(6):893-897. doi:10.1037/0022-006X.56.6.893

40. Kamaradova D, Prasko J, Latalova K, et al. Psychometric properties of the Czech version of the Beck Anxiety Inventory - comparison between diagnostic groups.. Neuro Endocrinol Lett. 2015;36 (7): 706-712.

41. Beck AT, Steer RA, Ball R, Ranieri W. Comparison of Beck Depression Inventories-IA and-II in Psychiatric Outpatients. $J$ Pers Assess. 1996;67(3):588-597. doi:10.1207/s15327752jpa6703_13

42. Ociskova M, Prasko J, Kupka M, et al. Psychometric evaluation of the Czech Beck Depression Inventory-II in a sample of depressed patients and healthy controls.. Neuro Endocrinol Lett. 2017;38 (2):98-106.

43. Carlson EB, Putnam FW. An update on the Dissociative Experience Scale: an update on the Dissociative. Dissociation. 1993;6:16-27. doi: $10.1177 / 1073191116645904$

44. Ptacek R, Bob P, Paclt I. Skala disociativnich zkusenosti - ceska verze [Dissociative Experiences Scale - A Czech Version]. Cesk Psychol. 2006;50(3):262-272.

45. Prasko J, Kamaradova D, Cakirpaloglu S, et al. Predicting the therapeutic response to intensive psychotherapeutic programs in patients with neurotic spectrum disorders. Act Nervosa Superior Rediviva. 2015;57(1-2):30-39.

46. Faul F, Erdfelder E, Lang A-G, Buchner A. G*Power 3: A flexible statistical power analysis program for the social, behavioral, and biomedical sciences. Behav Res Methods. 2007;39(2):175-191. doi:10.3758/bf03193146

47. Cohen J. Statistical Power Analysis for the Behavioral Sciences. 2nd ed. Hillsdale: Lawrence Erlbaum Associates; 1988. 
48. European Medicines Agency. Guideline for good clinical practice E6 (R2) (2018). http://www.ema.europa.eu/docs/en_GB/document library/Scientific_guideline/2009/09/WC500002874.pdf. Accessed 3 August 2020..

49. Lochner C, Stein DJ. Gender in obsessive-compulsive disorder and obsessive-compulsive spectrum disorders. Arch Women's Mental Health. 2001;4(1):19-26. doi:10.1007/s007370170004

50. Brakoulias V, Starcevic V, Belloch A, et al. Comorbidity, age of onset and suicidality in obsessive-compulsive disorder (OCD): an international collaboration. Compr Psychiatry. 2017;76:79-86. doi:10.1016/ j.comppsych.2017.04.002

51. Melca IA, Yücel M, Mendlowicz MV, de Oliveira-souza R, Fontenelle LF. The correlates of obsessive-compulsive, schizotypal, and borderline personality disorders in obsessive-compulsive disorder. $J$ Anxiety Disord. 2015;33:15-24. doi:10.1016/j. janxdis.2015.04.004

52. Jónsson H, Hougaard E. Group cognitive behavioural therapy for obsessive-compulsive disorder: A systematic review and meta-analysis. Acta Psychiatr Scand. 2009;119(2):98-106. doi:10.1111/j.1600-0447.2008.01270.x

53. Sunde T, Walseth LT, Himle JA, et al. A long-term follow-up of group behavioral therapy for obsessive-compulsive disorder in a general outpatient clinic in Norway. $J$ Obsessive Compuls Relat Disord. 2017;14:59-64. doi:10.1016/j.jocrd.2017.06.002

54. Prasko J, Grambal A, Kasalova P, et al. Impact of dissociation on treatment of depressive and anxiety spectrum disorders with and without personality disorders. Neuropsychiatric Dis Treat. 2016;12:2659-2676. doi:10.2147/NDT.S118058

55. Tatlı M, Cetinkaya O, Maner F. Evaluation of relationship between obsessive-compulsive disorder and dissociative experiences. Clin Psychopharmacol Neurosci. 2018;16(2):161-167. doi:10.9758/ cpn.2018.16.2.161
56. Belli H. Dissociative symptoms and dissociative disorders comorbidity in obsessive compulsive disorder: symptom screening, diagnostic tools and reflections on treatment. World J Clin Cases. 2014;2 (8):327-331. doi:10.12998/wjcc.v2.i8.327

57. Taylor S, Cox BJ. An expanded Anxiety Sensitivity Index: evidence for a hierarchic structure in a clinical sample. $J$ Anxiety Disord. 1998;12(5):463-483. doi:10.1016/s0887-6185(98)00028-0

58. Robinson LJ, Freeston MH. Emotion and internal experience in Obsessive Compulsive Disorder: reviewing the role of alexithymia, anxiety sensitivity and distress tolerance. Clin Psychol Rev. 2014;34 (3):256-271. doi:10.1016/j.cpr.2014.03.003

59. Rüsch N, Lieb K, Göttler I, et al. Shame and implicit self-concept in women with borderline personality disorder. Am J Psychiatry. 2007;164(3):500-508. doi:10.1176/ajp.2007.164.3.500

60. Thiese MS, Ronna B, Ott U. P value interpretations and considerations. J Thorac Dis. 2016;8(9):E928-E931. doi:10.21037/ jtd.2016.08.16

61. Perez V, Barrachina J, Soler J, et al. The clinical global impression scale for borderline personality disorder patients (CGI-BPD): a scale sensible to detect changes.. Actas Esp Psiquiatr. 2007;35(4):229-235.

62. Alreja S, Sengar S, Singh A, Mishra D. Insight and its relationship with stigma in psychiatric patients. Industrial Psychiatry J. 2009;18 (1):39-42. doi:10.4103/0972-6748.57858

63. Lien Y-J, Chang H-A, Kao Y-C, Tzeng N-S, Lu C-W, Loh C-H. Insight, self-stigma and psychosocial outcomes in Schizophrenia: a structural equation modelling approach. Epidemiol Psychiatr Sci. 2018;27(2):176-185. doi:10.1017/S2045796016000950
Psychology Research and Behavior Management

\section{Publish your work in this journal}

Psychology Research and Behavior Management is an international, peer-reviewed, open access journal focusing on the science of psychology and its application in behavior management to develop improved outcomes in the clinical, educational, sports and business arenas. Specific topics covered in the journal include: Neuroscience, memory and decision making; Behavior modification and management; Clinica applications; Business and sports performance management; Social and developmental studies; Animal studies. The manuscript management system is completely online and includes a very quick and fair peer-review system, which is all easy to use. Visit http://www. dovepress.com/testimonials.php to read real quotes from published authors. 